\title{
Quantum Computing Arrays Made of Two Types of Atom
}

\author{
Two research teams have created arrays containing two different neutral \\ atoms, a promising platform for quantum computing. \\ By Sophia Chen
}

1 sing arrays of a single type of neutral atom, researchers have recently demonstrated that they can orchestrate atomic interactions for applications such as quantum computing or the controlled formation of single molecules. They are now looking to create neutral-atom arrays from multiple atomic species, something that could enable more advanced quantum computing protocols, for example. Toward that goal, Xiaodong He of the Wuhan Institute of Physics and Mathematics, China, and colleagues have now experimentally demonstrated how to produce arrays containing two different rubidium isotopes [1]. The demonstration parallels another by a team led by Hannes Bernien at the University of Chicago, which realized arrays of rubidium and cesium atoms [2].

Both teams used optical tweezers to arrange their atom arrays. He and his colleagues worked with two isotopes of rubidium. They loaded a mixture of around 30 rubidium-85 $\left({ }^{85} \mathrm{Rb}\right)$ and rubidium-87 ( $\left.{ }^{87} \mathrm{Rb}\right)$ atoms into optical tweezer arrays from a magneto-optical trap. They then used fluorescence imaging to measure the initial array pattern that the atoms formed. Next,
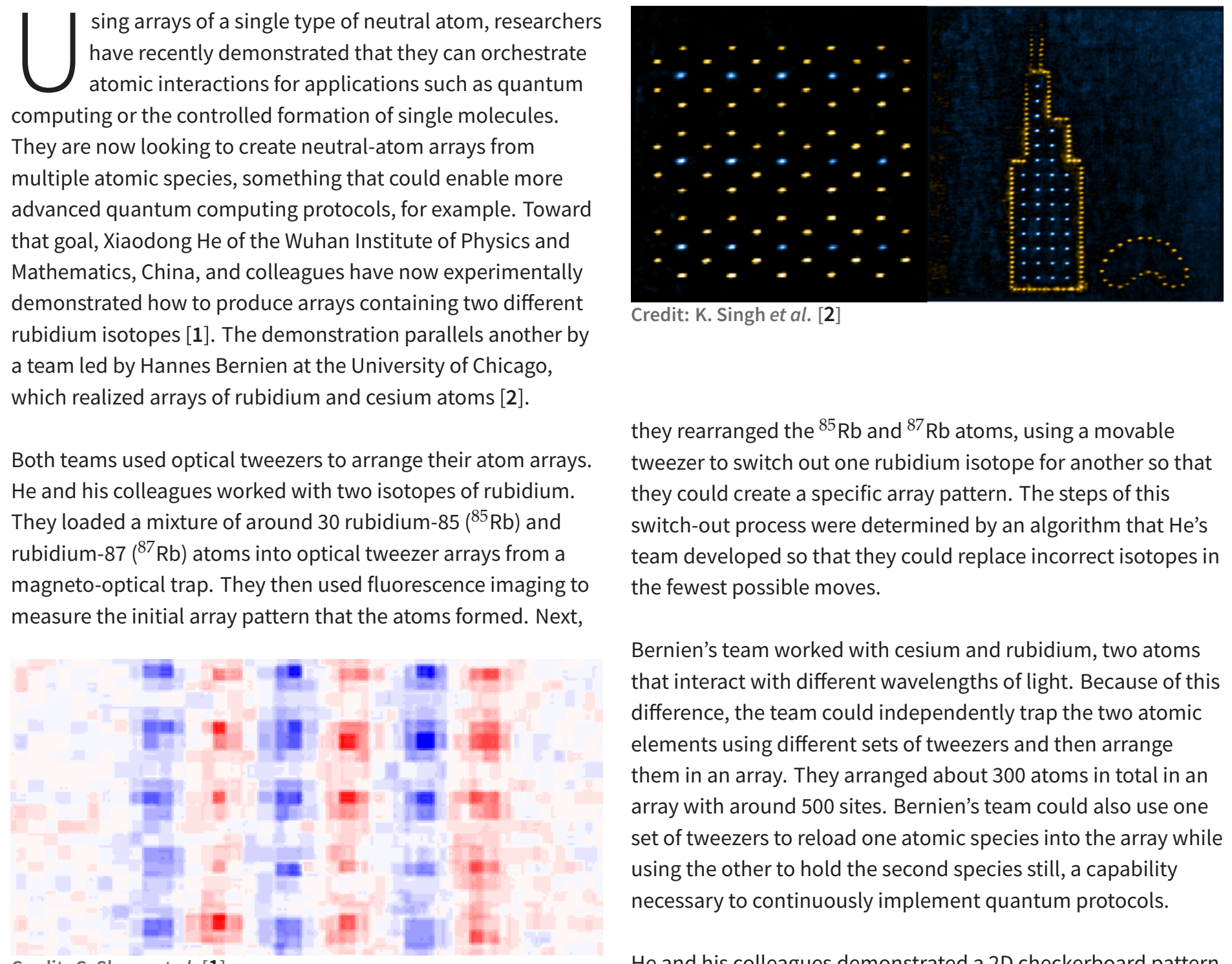

Credit: K. Singh et al. [2]

they rearranged the ${ }^{85} \mathrm{Rb}$ and ${ }^{87} \mathrm{Rb}$ atoms, using a movable tweezer to switch out one rubidium isotope for another so that they could create a specific array pattern. The steps of this switch-out process were determined by an algorithm that He's team developed so that they could replace incorrect isotopes in the fewest possible moves.

Bernien's team worked with cesium and rubidium, two atoms that interact with different wavelengths of light. Because of this difference, the team could independently trap the two atomic elements using different sets of tweezers and then arrange them in an array. They arranged about 300 atoms in total in an array with around 500 sites. Bernien's team could also use one set of tweezers to reload one atomic species into the array while using the other to hold the second species still, a capability necessary to continuously implement quantum protocols.

He and his colleagues demonstrated a 2D checkerboard pattern of alternating isotopes, which they say is well suited for executing some types of quantum error correction code. They 
also made a 2D striped pattern. Bernien's team also created various $2 \mathrm{D}$ patterns, including an arrangement that resembled the outline of the Sears Tower. In future work, both teams plan to use their arrays to demonstrate quantum computing protocols.

Sophia Chen is a freelance science writer based in Columbus, Ohio.

\section{REFERENCES}

1. C. Sheng et al., "Defect-free arbitrary-geometry assembly of mixed-species atom arrays," Phys. Rev. Lett. 128, 083202 (2022).

2. K. Singh et al., "Dual-element, two-dimensional atom array with continuous-mode operation,” Phys. Rev. X 12, 011040 (2022). 